\title{
A Network Pharmacology Approach to Predict the Proangiogenesis Mechanism of Huangqi-Honghua Herb Pair after Cerebral Ischemia
}

\author{
Jinyi Cao $\mathbb{D}^{1},{ }^{1}$ Lu Lei, ${ }^{2}$ Kai Wang, ${ }^{3}$ Jing Sun, ${ }^{4}$ Yi Qiao, ${ }^{1}$ Jialin Duan, ${ }^{1}$ Chao Zhao, ${ }^{1}$ Jia Cui $\left(D,{ }^{1}\right.$ \\ Zhijun Feng, ${ }^{1}$ Jing-wen Wang $\mathbb{D},{ }^{1}$ Aidong Wen, ${ }^{1}$ and Zhifu Yang $\mathbb{D}^{1}$ \\ ${ }^{1}$ Department of Pharmacy, Xijing Hospital, Fourth Military Medical University, Xi'an 710032, China \\ ${ }^{2}$ School of Basic Medicine and Clinical Pharmacy, China Pharmaceutical University, Nanjing 211198, China \\ ${ }^{3}$ College of Pharmacy, Shaanxi University of Chinese Medicine, Xianyan 712046, China \\ ${ }^{4}$ Department of Anesthesiology, Northwest Women's and Children's Hospital, Xi'an 710061, China
}

Correspondence should be addressed to Jing-wen Wang; wangjingwen8021@163.com and Zhifu Yang; yangtian_1973@163.com

Received 27 July 2020; Revised 25 March 2021; Accepted 8 April 2021; Published 16 April 2021

Academic Editor: Nguyen Phuoc Long

Copyright (c) 2021 Jinyi Cao et al. This is an open access article distributed under the Creative Commons Attribution License, which permits unrestricted use, distribution, and reproduction in any medium, provided the original work is properly cited.

\begin{abstract}
Objective. Huangqi-Honghua herb pair is known for its medicinal value to treat Qi deficiency and blood stasis syndrome with a long history in clinical practice. To understand its possible mechanism in a systematic study, a network pharmacological method was addressed. Methods. Detailed information on the $\mathrm{HH}$ compounds was obtained from two public databases, and oral bioavailability (OB) and drug-like (DL) of the compounds were evaluated. A correlation between $\mathrm{HH}$ compounds, its potential targets, and known targets was extrapolated, and the herb-compound-target-disease (H-C-T-D) network was established. Next, the pathway enrichment and essential genes were analyzed. Then, three key genes (VEGFA, VEGFR2, and eNOS), highly associated with angiogenesis, were screened and verified through western blot assay. Results. Out of $276 \mathrm{compounds,} 21 \mathrm{HH}$ compounds and 78 target genes regulating the major pathways associated with CI in the network are analyzed. The bioactive compounds in $\mathrm{HH}$ were active in various signal transduction pathways such as the toll-like receptor signaling pathway, VEGF signaling pathway, TNF signaling pathway, and HIF-1 signaling pathway are important pathways that may regulate anti-inflammatory, antiapoptotic, immune correlation, and antioxidative effects. The core genes are PTGS2, TNF, NOS2, IL6, BCL2, IL1B, SOD2, NOS3, SOD1, MMP9, and VEGFA. The in vitro results suggested that HH treatment could significantly elevate the expression of proangiogenic genes such as VEGFA, VEGFR2, and eNOS compared with OGD groups. Conclusions. Our results predict that HH may regulate the expression of VEGFA, VEGFR2, and eNOS via the VEGF and HIF-1 signaling pathway to promote angiogenesis and alleviate cerebral ischemia injury.
\end{abstract}

\section{Introduction}

Ischemic stroke (cerebral ischemia, CI) has become a major public health concern with morbidity, mortality, and health care costs [1]. Irrespective of the current standard of care options (e.g., mechanical or pharmacological (tissue plasminogen activator, $\mathrm{PAA}$ ) reperfusion) available in the clinical practice, there is an unmet need for ischemic stroke because of the narrow treatment time window [2]. Thus, there is an unmet need for developing regenerative medicine to address
$\mathrm{CI}$ and its secondary effects. One of the devastating consequences of CI is decreased blood supply to the injured brain, and it leads to the functional impairment of the brain tissue in the affected area. Developing an effective strategy to increase the blood supply to the ischemic area may potentially rescue the brain tissue from death and may improve the recovery rate of the patients [3]. Previous studies have indicated that the higher the vascular density of ischemic penumbra, the longer the survival time of ischemic stroke patients $[4,5]$. Therefore, proangiogenesis may be a valid 
antistroke strategy to address current clinical hurdles associated with a stroke patient, which has the potential to repair and rewire the injured nerve connections.

Traditional Chinese medicine (TCM) has been shown to be beneficial in promoting developmental angiogenesis, which highlights "reinforcing qi to enrich the blood, dispelling stasis to promote regeneration" theory by associating with angiogenesis together. Radix Astragali (Huangqi) is the dry root of Astragalus membranaceus (Fisch.) Bge, which has been commonly used in patients with stroke or chronic weakness in China because it can enhance Qi and dispel blood stasis, with antioxidation, anti-inflammatory, immunomodulation, and anticancer effects in modern medical research [6, 7]. Carthamus tinctorius L. (Honghua), a dried flower, often used to promote blood circulation, dispel blood stasis, and relieve pain [8, 9]. Huangqi-Honghua $(\mathrm{HH})$ herb pair has been used widely in clinical practice for treating Qi deficiency and blood stasis syndrome, especially cerebrovascular and cardiovascular diseases, with a long history, such as Buyang Huanwu decoction, a wellknown TCM formula [10-12]. In our previous study, by using a rat model of middle cerebral artery occlusion (MCAO), we have validated the synergetic effect of hydroxysafflor yellow A and astragaloside IV (main constituents of $\mathrm{HH}$ ) in removing blood stasis (QDBS) syndrome [13].

Network pharmacology follows the dogma of "drugtargets-gene-disease," to predict drug targets and improve the efficiency of drug discovery effort. Network pharmacology maintains integrity and systematic characteristic, similar to the principle of traditional Chinese medicine that involves multiple components and multiple targets [14]. Network pharmacology has become a novel and efficient way to interpret the complex pharmacological mechanism of TCMs scientifically, particularly, formulae orchestration [15].

Despite a few studies that have proved the protective effect of $\mathrm{HH}$ in the cerebral hemisphere, pharmacologybased prediction of complete profiling of bioactive components and the network of their target pathways has not fully been elucidated. Thus, in the present study, we have explored the proangiogenesis mechanisms of $\mathrm{HH}$ in the etiology of CI. In this study, we have established the herbcompound-target-disease (H-C-T-D) networks by utilizing the network pharmacology approach. Furthermore, to demonstrate angiogenesis, one key pathway of $\mathrm{HH}$ in cerebral protection, we have identified three key targets, which is highly associated with angiogenesis, through immunoblot assay. The workflow is shown in Figure 1.

\section{Materials and Methods}

2.1. Chemical Databases. Information on the herbs and compounds related to $\mathrm{HH}$ were collected from two botanical chemistry databases: TCM Database of @Taiwan (http://tcm.cmu.edu.tw/) and the traditional Chinese Medicine system Pharmacology Database (TCMSP, http://ibts.hkbu. edu.hk/LSP/tcmsp.php). The chemical structures, 2D structure, molecular weight, and chemical number are reported on ChEMBL (http://www.ebi.ac.uk/chembl/) and NCBI PubChem (http://puchem.nvbi.nlm.nih.gov/).

2.2. Oral Bioavailability and Drug-Likeness of $\mathrm{HH}$ Components. All compounds in $\mathrm{HH}$ were evaluated for their oral bioavailability (OB) and drug-likeness (DL). OB is one of the most common pharmacokinetic parameters in the drug screening process, which explains the percentage of an oral drug that remains unchanged after entering into the systemic circulation. In addition, $\mathrm{OB}$ provides a clue for the fusion of the ADME process. DL measures the extent of the "drug-like" property of a compound and determines whether the compound influences absorption, distribution, metabolism, and excretion (ADME) in the human body like an approved drug. In this study, chemicals only with an $\mathrm{OB}$ $\geq 30$ and DL $\geq 0.18$ (recommended by TCMSP database) were considered for further valuation [16].

2.3. Collection Target Genes Relevant to the Screened Compound. UniProt (http://www.uniprot.org/) platform, by setting the filter to "Homo sapiens," was used to identify the target genes, closely relevant to chemicals. Meanwhile, information on target genes related to the CI was obtained from the therapeutic target database (TTD; http://www. bidd.Nus.edu.sg/group/cjttd/TTD\%20HOME.asp) and DisGeNETv6.0 (http://www.disgenet.org/web/DisGeNET/ menu/home). Finally, we assigned the gene names and ID by constructing herbs-ingredients-targets relationship dataset for $\mathrm{HH}$.

2.4. Network Construction and Pathway Analyses. The herbcompound-target-disease (H-C-T-D) networks were established using Cytoscape 3.3.0, JAVA software. Functional pathways annotation related to CI and enrichment evaluation was evaluated using the Kyoto Encyclopedia of Genes (KEGG), Genomes (GO), and the Database for Annotation, Visualization, and Integrated Discovery (DAVID) version 6.8 (http://david.ncifcrf.gov/).

2.5. Protein-Protein Interaction (PPI) Data. PPI data were collected from the Search Tool for the Retrieval of Interacting Genes (STRING) database (http://string-db.org/, 11.0 version), and possible protein-protein interactions were predicted. We chose and showed the top 20 target genes with confidence score $>0.9$ as the core genes for CI.

\subsection{Pharmacological Experiment}

2.6.1. Materials. $\mathrm{HH}$ granules (Huangqi: Honghua $=5: 1$, previous research has confirmed) were obtained from Guangdong Yifang Pharmaceuticals Co., Ltd. (product batch: 20170606). The rat brain microvascular endothelial cells (BMECs) were purchased from the Cell Biologics Company (\#C57-6023, Chicago, IL). Primary antibodies against $\beta$-actin, VEGFA, eNOS, MMP9, IL6, and secondary antibody were all purchased from Abcam Technology 


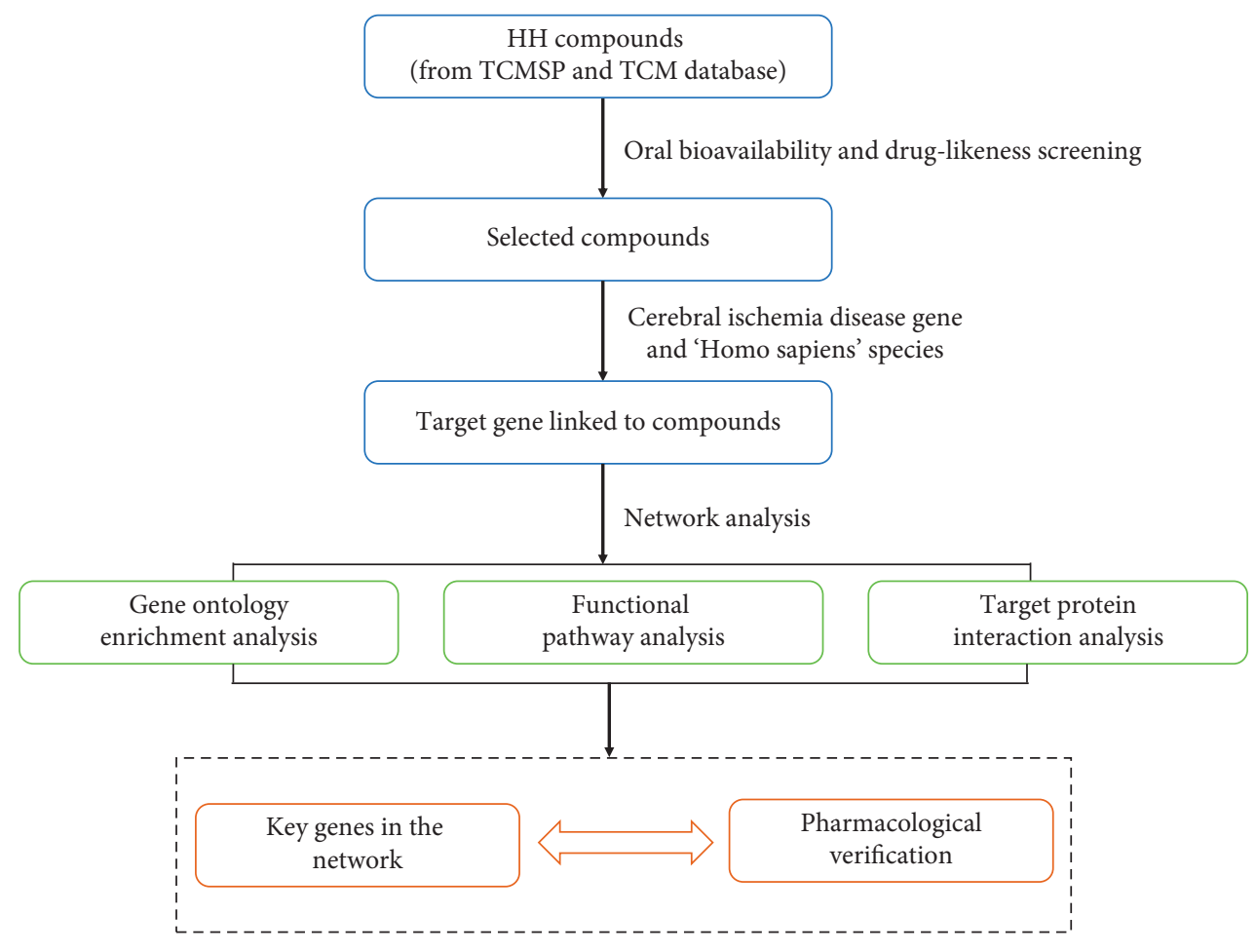

FIgURE 1: Workflow of network pharmacology analysis.

(Cambridge, UK). All other reagents are analytical reagents and commercially available.

2.6.2. Cell Culture and Treatments. Cells were divided into three groups: control group, OGD group, and OGD group treated with $\mathrm{HH}(100 \mu \mathrm{g} / \mathrm{mL})$. Oxygen-glucose deprivation (OGD) was established as follows: cells were rinsed once with glucose-free DMEM (Gibco, Rockville, MD) and transferred to an anaerobic chamber (Forma Scientific, Waltham, MA) containing a gas mixture composed of $7 \% \mathrm{CO}_{2}$ and $93 \% \mathrm{~N}_{2}$ for $6 \mathrm{~h}$ at $37^{\circ} \mathrm{C}$. Then, the cells were returned to the normal culture condition. Control BMECs were cultured in complete DMEM under normal conditions. $\mathrm{HH}$ groups were treated with $\mathrm{HH}$ (dissolved in complete DMEM and filtered with a $0.22 \mu \mathrm{m}$ membrane filter) for $12 \mathrm{~h}$. The control group was treated without $\mathrm{HH}$.

2.6.3. Western Blot Assay. BMECs were lysed with cold RIPA buffer (Rockford, IL, USA) for $30 \mathrm{~min}$. The whole cell lysates were separated by $10 \%$ sepharose gel, and PVDF membranes (Millipore, USA) were used to transfer protein. The membranes were incubated with $5 \%$ bovine serum albumin (BSA) and overnight with primary antibodies against VEGFA (1:1000, rabbit), VEGFR2 (1:1000, rabbit), and eNOS $\left(1: 1000\right.$, rabbit) at $4^{\circ} \mathrm{C}$. After that, membranes were incubated with secondary antibody at a $1: 5000$ dilution at $37^{\circ} \mathrm{C}$ for $1 \mathrm{~h}$. After ECL-Plus reagent (Santa Cruz, USA) treatment, the blots were analyzed with Quantity One System image analysis software (Bio-Rad, USA).
2.6.4. Statistical Analysis. The results were expressed as mean \pm standard deviation (S.D.) and analyzed with oneway analysis of variance (ANOVA), followed by a Tukey's post hoc test was used for analyzing differences between groups. $p<0.05$ presented statistical significance.

\section{Results}

3.1. Identification of Active Compounds in $\mathrm{HH}$. $\mathrm{HH}$ consists of a total of 276 compounds, 87 in Astragalus (Huangqi) and 189 in Safflower (Honghua). All 276 compounds were screened with $\mathrm{OB}$ and DL, out of which, only 42 compounds were recommended for the final screening. The remaining compounds were discarded. The therapeutic effect of a few selected compounds on CI was confirmed through the related literature search. Therefore, preselected active ingredients were added manually, including hydroxysafflor yellow A and astragaloside IV [17-19]. After that, the repeated ingredients and the ingredients with the unclear target are deleted. The selected 27 compounds from $\mathrm{HH}$ is shown in Table 1 and Figure 2(a).

3.2. Compounds-Targets Analysis. The active ingredients of $\mathrm{HH}$ obtained from the screening assay were predicted using TCMSP and CTD database (https://ctdbase.org/). The repeated targets were deleted, and the unclear targets were eliminated. A total of 459 predicted target genes were obtained related to the 27 identified compounds in $\mathrm{HH}$ (Figures 2(a) and 2(c)). The compound-target gene network includes 683 nodes and 1146 edges as shown in Figure 2(e). The nodes (the points of communication or redistribution) and edges (the lines of communication joining the nodes) of 
TABle 1: A list of the final selected compounds among the two herbal medicines for network analysis.

\begin{tabular}{lcccl}
\hline No. & Compound & OB (\%) & DL & Herb \\
\hline 1 & Kaempferol & 41.88 & 0.24 & $\begin{array}{l}\text { Safflower, } \\
\text { astragalus }\end{array}$ \\
& & & & Safflower, \\
2 & Quercetin & 46.43 & 0.28 & astragalus \\
3 & Lignan & 43.32 & 0.65 & Safflower \\
4 & Phytoene & 39.56 & 0.5 & Safflower \\
5 & Phytofluene & 43.18 & 0.5 & Safflower \\
6 & Pyrethrin II & 48.36 & 0.35 & Safflower \\
7 & 6-Hydroxykaempferol & 62.13 & 0.27 & Safflower \\
8 & Baicalein & 33.52 & 0.21 & Safflower \\
9 & Qt_carthamone & 51.03 & 0.2 & Safflower \\
10 & Quercetagetin & 45.01 & 0.31 & Safflower \\
11 & Beta-carotene & 37.18 & 0.58 & Safflower \\
12 & Baicalin & 40.12 & 0.75 & Safflower \\
13 & Beta-sitosterol & 36.91 & 0.75 & Safflower \\
14 & Poriferast-5-en-3beta-ol & 36.91 & 0.75 & Safflower \\
15 & Stigmasterol & 43.83 & 0.76 & Safflower \\
16 & Luteolin & 36.16 & 0.25 & Safflower \\
17 & CLR & 37.87 & 0.68 & Safflower \\
18 & Hydroxysafflor yellow A & 4.77 & 0.68 & Safflower \\
19 & Mairin & 55.38 & 0.78 & Astragalus \\
20 & Jaranol & 50.83 & 0.29 & Astragalus \\
21 & Hederagenin & 36.91 & 0.75 & Astragalus \\
22 & Isorhamnetin & 49.6 & 0.31 & Astragalus \\
23 & Bifendate & 31.1 & 0.67 & Astragalus \\
24 & Formononetin & 69.67 & 0.21 & Astragalus \\
25 & Isoflavanone & 109.99 & 0.3 & Astragalus \\
26 & Calycosin & 47.75 & 0.24 & Astragalus \\
27 & Astragaloside IV & 17.74 & 0.15 & Astragalus \\
\hline & & & & \\
\hline & & &
\end{tabular}

$\mathrm{HH}$ are listed in Table 2. Kaempferol is the compound of maximum interactions with target genes.

3.3. Network Construction and Analysis. IOut of the 459 matching $\mathrm{HH}$-associated genes and $274 \mathrm{CI}$-related genes, we streamlined 78 overlapping genes while constructing the gene network (Figure 2(b), Table 3). This gene network included 101 nodes (2 Chinese herb medicines, 21 compounds, and 78 target genes) and 367 edges (Figure 3), and 21 compounds were categorized as flavonoids, lignans, terpene, sterols, triterpenoids, and their glycosides. Particularly, 13 compounds (astragaloside IV, calycosin, formononetin, bifendate, isorhamnetin, hydroxysafflor yellow A, quercetin, luteolin, kaempferol, $\beta$-sitosterol, baicalin, $\beta$-carotene, and pyrethrin II) were related to more than five genes, and 18 genes (PTGS2, TNF, CASP3, NOS2, RELA, PPARG, IL6, BCL2, NFKBIA, IL1B, CAT, MAPK1, CASP9, MAPK3, TP53, PARP1, SOD2, NOS3, NFE2L2, SOD1, MMP9, ICAM1, JUN, and VEGFA) related to more than five compounds. Therefore, we speculate that these compounds and genes might be key nodes in this network. The compound-target gene network will be conducive to interpretation and prediction the "multicompound, multitarget, and multipathway" of $\mathrm{HH}$.

3.4. Pathway and GO Term Enrichment Analysis. To explore the signaling pathway and functions of the identified target genes, 78 candidate targets were analyzed by GO and enriched by the KEGG pathway. The top 6 enriched conditions were found to be involved in the biological process (BP), cell component (CC), and molecular function (MF) (Figure 4(a)). According to the results of GO enrichment analysis, the positive regulation of transcription from RNA polymerase II promoter, inflammatory response, response to lipopolysaccharide, aging, response to drug, signal transduction etc., dominates the biological process. Forty-two targets, the largest proportion, account for cell components. Screening of $p<0.05$ signal pathway by KEGG enrichment analysis eliminates signaling pathways that are not associated with CI and get the top 18 signaling pathways (Figure 4(b) and Table 4). Therefore, we speculate that the TNF signaling pathway, toll-like receptor signaling pathway, HIF-1 signaling pathway, PI3K-Akt signaling pathway, NFkappa B signaling pathway, and VEGF signaling pathway may be the important pathways to exert their synergistic effects against cerebral ischemia injury.

3.5. PPI Networks. In the PPI relationships network, we found 78 nodes and 1429 edges (Figure 5(a)). The top 20 core genes, including tumor necrosis factor (TNF), interleukin 6 (IL6), MAP kinase-activated protein kinase 3 (MAPK3), vascular endothelial growth factor A (VEGFA), interleukin 10 (IL10), matrix metalloproteinase-9 (MMP9), and endothelial nitric oxide synthase (eNOS/NOS3) were selected (Figure 5(b)). Darker color scheme denotes a higher score.

3.6. Experimental Validation. To confirm the results from the network and to verify that angiogenesis is indeed one of the key pathways of $\mathrm{HH}$ in cerebral protection, we selected three targets related to angiogenesis (VEGFA, VEGFR2, and eNOS) for pharmacological validation (Figure 6). Western blot analysis indicated that treatment with $\mathrm{HH}$ could significantly increase the expression of VEGFA, VEGFR2, and eNOS, compared with the OGD group $(* p<0.05$, $* * p<0.01$ compared with control group, and $\# p<0.05$ compared with OGD group).

\section{Discussion}

Multicompound TCMs, with multiple biological targets, are the prime focus in Chinese clinical practice for more than thousand years. Surprisingly, TCMs are known to target multiple biological pathways to defend against diseases. However, the traditional method of usage of TCMs barely provides insights into the biological complexity of compounds, its biological targets, and associated disease, which limits the development and reformulation of TCMs. Lately, network pharmacology approach of analyzing TCMs gains more popularity that dissects the pharmacological mechanism of action [20-22].

In the current study, a network pharmacology analysis of $\mathrm{HH}$ identified 2 herbs, 21 compounds, as well as 78 target gene-regulated major pathways associated with CI. Through the pathway enrichment analysis, we found that the targets of active ingredients in $\mathrm{HH}$ against cerebral ischemia injury 


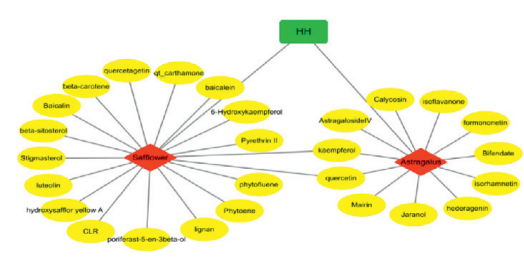

(a)

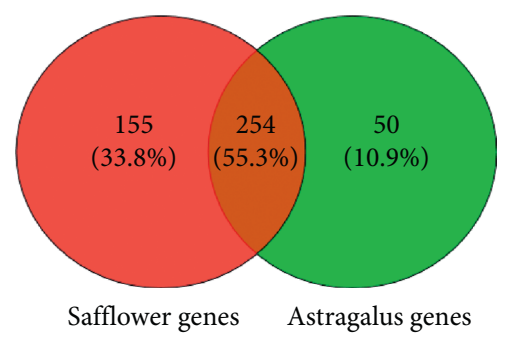

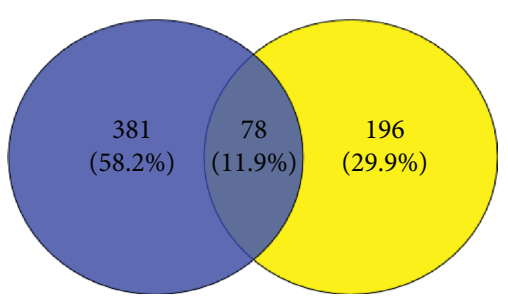

Compound genes

(b)

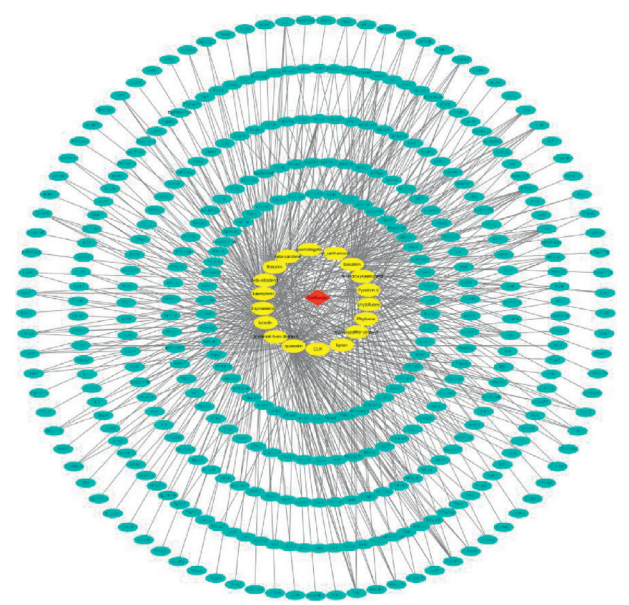

(d)

(c)

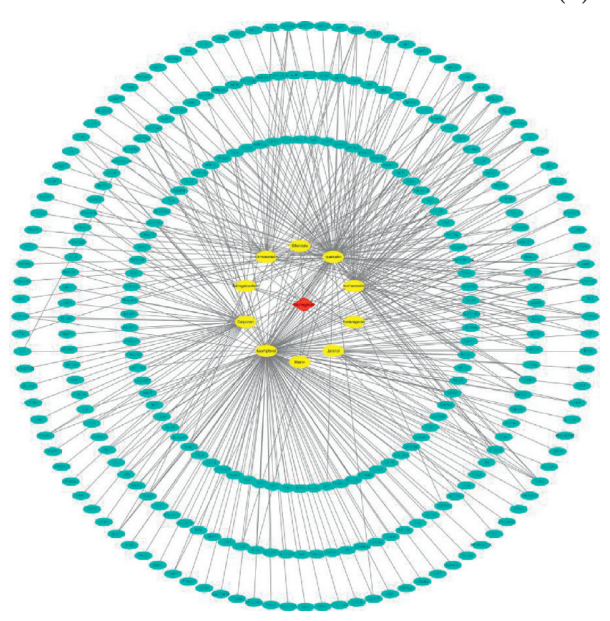

(e)

Figure 2: Linkage of target compounds and target genes. (a) The network of herbal medicine-compound in HH. (b) The Venn diagram of the compound genes and disease genes. (c) The Venn diagram of the target genes for safflower (Honghua) and astragalus (Huangqi). (d, e) The pharmacology networks of two herb medicines (red diamonds) which connect with target genes (blue ellipses) compounds (yellow ellipses).

TABLe 2: Nodes and edges of HH.

\begin{tabular}{lcc}
\hline & Safflower & Astragalus \\
\hline Compounds & 18 & 11 \\
Targets of compounds & 409 & 304 \\
Nodes & 391 & 292 \\
Edges & 680 & 466 \\
Number of maximum interactions & 159 & 159 \\
Compounds of maximum interactions with target genes & Kaempferol & Kaempferol \\
\hline
\end{tabular}


TABle 3: A list of the compound and disease common target protein.

\begin{tabular}{|c|c|c|}
\hline No. & Gene & Compounds \\
\hline 1 & PTGS2 & $\begin{array}{l}\text { Lignan, pyrethrin II, 6-hydroxykaempferol, baicalein, qt_carthamone, quercetagetin, baicalin, kaempferol, luteolin, } \\
\text { quercetin, jaranol, isorhamnetin, bifendate }\end{array}$ \\
\hline 2 & TNF & $\begin{array}{c}\text { Baicalein, beta-carotene, baicalin, kaempferol, luteolin, quercetin, hydroxysafflor yellow A, isorhamnetin, bifendate, } \\
\text { Astragaloside IV, stigmasterol }\end{array}$ \\
\hline 3 & CASP3 & $\begin{array}{c}\text { Baicalein, beta-carotene, baicalin, beta-sitosterol, kaempferol, luteolin, quercetin, isorhamnetin, formononetin, } \\
\text { astragaloside IV }\end{array}$ \\
\hline 4 & NOS2 & 6-Hydroxykaempferol, baicalein, baicalin, kaempferol, luteolin, quercetin, jaranol, isorhamnetin, bifendate \\
\hline 5 & RELA & Safflower, baicalein, baicalin, kaempferol, luteolin, quercetin, isorhamnetin, bifendate, astragaloside IV \\
\hline 6 & PPARG & $\begin{array}{l}\text { 6-Hydroxykaempferol, baicalein, quercetagetin, baicalin, kaempferol, quercetin, hydroxysafflor yellow A, isorhamnetin, } \\
\text { formononetin }\end{array}$ \\
\hline 7 & IL6 & Baicalin, kaempferol, luteolin, quercetin, hydroxysafflor yellow A, isorhamnetin, bifendate, astragaloside IV \\
\hline 8 & BCL2 & Baicalein, beta-carotene, beta-sitosterol, kaempferol, luteolin, quercetin, isorhamnetin, formononetin \\
\hline 9 & NFKBIA & Baicalein, baicalin, kaempferol, luteolin, quercetin, isorhamnetin, formononetin, astragaloside IV \\
\hline 10 & IL1B & Baicalein, beta-carotene, baicalin, kaempferol, luteolin, quercetin, isorhamnetin, astragaloside IV \\
\hline 11 & CAT & Beta-carotene, beta-sitosterol, kaempferol, luteolin, quercetin, isorhamnetin, bifendate \\
\hline 12 & MAPK1 & Calycosin, beta-carotene, baicalein, luteolin, kaempferol, quercetin \\
\hline 13 & CASP9 & Beta-sitosterol, kaempferol, luteolin, quercetin, isorhamnetin, formononetin \\
\hline 14 & MAPK3 & Baicalein, beta-carotene, kaempferol, luteolin, quercetin, calycosin \\
\hline 15 & TP53 & Baicalin, luteolin, quercetin, formononetin, kaempferol, beta-carotene \\
\hline 16 & PARP1 & Beta-sitosterol, kaempferol, luteolin, quercetin, isorhamnetin \\
\hline 17 & JUN & Baicalein, beta-carotene, kaempferol, luteolin, quercetin \\
\hline 18 & VEGFA & Baicalein, kaempferol, luteolin, quercetin, calycosin \\
\hline 19 & NFE2L2 & Kaempferol, luteolin, quercetin, astragaloside IV \\
\hline 20 & SOD1 & Kaempferol, luteolin, quercetin, astragaloside IV \\
\hline 21 & MMP9 & Baicalein, luteolin, quercetin, hydroxysafflor yellow A \\
\hline 22 & ICAM1 & Kaempferol, luteolin, quercetin, hydroxysafflor yellow A \\
\hline 23 & SOD2 & Beta-carotene, kaempferol, quercetin, astragaloside IV \\
\hline 24 & NOS3 & Luteolin, isorhamnetin, formononetin, quercetin \\
\hline 25 & CSF2 & Baicalein, kaempferol, luteolin, quercetin \\
\hline 26 & CYCS & Baicalein, beta-sitosterol, quercetin, kaempferol \\
\hline 27 & MPO & Baicalein, luteolin, quercetin, astragaloside IV \\
\hline 28 & HIF1A & Kaempferol, luteolin, quercetin, formononetin \\
\hline 29 & APP & Baicalein, kaempferol, quercetin \\
\hline 30 & IL10 & Stigmasterol, luteolin, bifendate \\
\hline 31 & CCL2 & Kaempferol, luteolin, quercetin \\
\hline 32 & FOS & Kaempferol, luteolin, quercetin \\
\hline 33 & NFKB1 & Baicalein, luteolin, quercetin \\
\hline 34 & STAT3 & Luteolin, baicalein, quercetin \\
\hline 35 & AGT & Isorhamnetin, quercetin \\
\hline 36 & $\mathrm{CD} 40$ & Luteolin, hydroxysafflor yellow A \\
\hline 37 & EGR1 & Luteolin, quercetin \\
\hline 38 & CXCL10 & Luteolin, quercetin \\
\hline 39 & SELE & Kaempferol, luteolin \\
\hline 40 & CEBPB & Baicalein, quercetin \\
\hline 41 & TLR4 & Baicalin, quercetin \\
\hline 42 & EPO & Formononetin, calycosin \\
\hline 43 & SERPINE1 & Isorhamnetin, quercetin \\
\hline 44 & JAK2 & Quercetin \\
\hline 45 & CCL3 & Isorhamnetin \\
\hline 46 & NR3C2 & CLR \\
\hline 47 & CALM1 & Lignan \\
\hline 48 & RB1 & Baicalein \\
\hline 49 & IGF1 & Baicalein \\
\hline 50 & APOE & Kaempferol \\
\hline 51 & $\mathrm{BBC} 3$ & Kaempferol \\
\hline 52 & ATM & Kaempferol \\
\hline 53 & CD14 & Kaempferol \\
\hline 54 & H2AFX & Kaempferol \\
\hline 55 & RIPK1 & Kaempferol \\
\hline 56 & VDR & Kaempferol \\
\hline
\end{tabular}


TABle 3: Continued.

\begin{tabular}{lcc}
\hline No. & Gene & Compounds \\
\hline 57 & PLAU & Baicalein \\
58 & ADIPOQ & Beta-carotene \\
59 & GOT1 & Baicalin \\
60 & PIK3CG & 6-Hydroxykaempferol \\
61 & HSPA1A & Quercetin \\
62 & FOSB & Luteolin \\
63 & GFAP & Luteolin \\
64 & GPX1 & Luteolin \\
65 & HBEGF & Luteolin \\
66 & IL17A & Luteolin \\
67 & JUNB & Luteolin \\
68 & JUND & Luteolin \\
69 & DDIT3 & Quercetin \\
70 & SIRT1 & Quercetin \\
71 & HMGB1 & Quercetin \\
72 & ALB & Quercetin \\
73 & PLAT & Quercetin \\
74 & CREB1 & Quercetin \\
75 & ACE & Luteolin \\
76 & AGER & Luteolin \\
77 & SP1 & Quercetin \\
78 & POU5F1 & Kaempferol \\
\hline
\end{tabular}

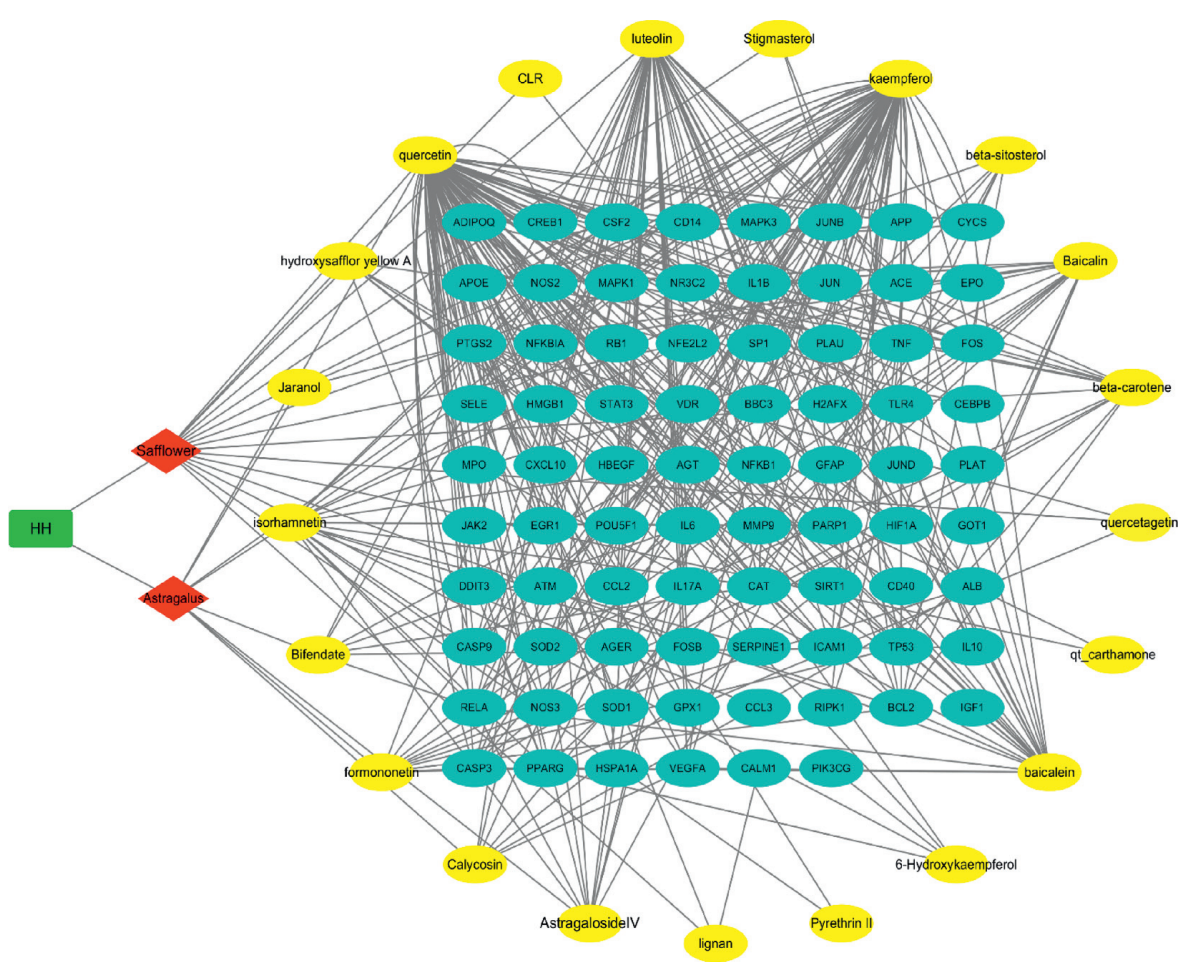

Figure 3: The herb-compound-gene network for $\mathrm{HH}$.

mainly participate in numerous signal transduction pathways such as TNF signaling pathway, toll-like receptor signaling pathway, HIF-1 signaling pathway, PI3K-Akt signaling pathway, and VEGF signaling pathway. These key pathways may regulate anti-inflammatory, antiapoptotic, immune correlation, and antioxidative effects. Furthermore, the PPI system analysis indicates these genes played vital roles in CI (Figure 5(b)), such as TNF, IL6, MAPK3, VEGFA, IL10, and MMP9. In addition, three key targets (VEGFA, VEGFR2, and eNOS), predicted in the network, highly related to angiogenesis were verified by using western blot assay.

According to the TCM theory, Qi and blood dysfunction is one key pathogenesis of CI. In the clinic, Qi-tonifying 

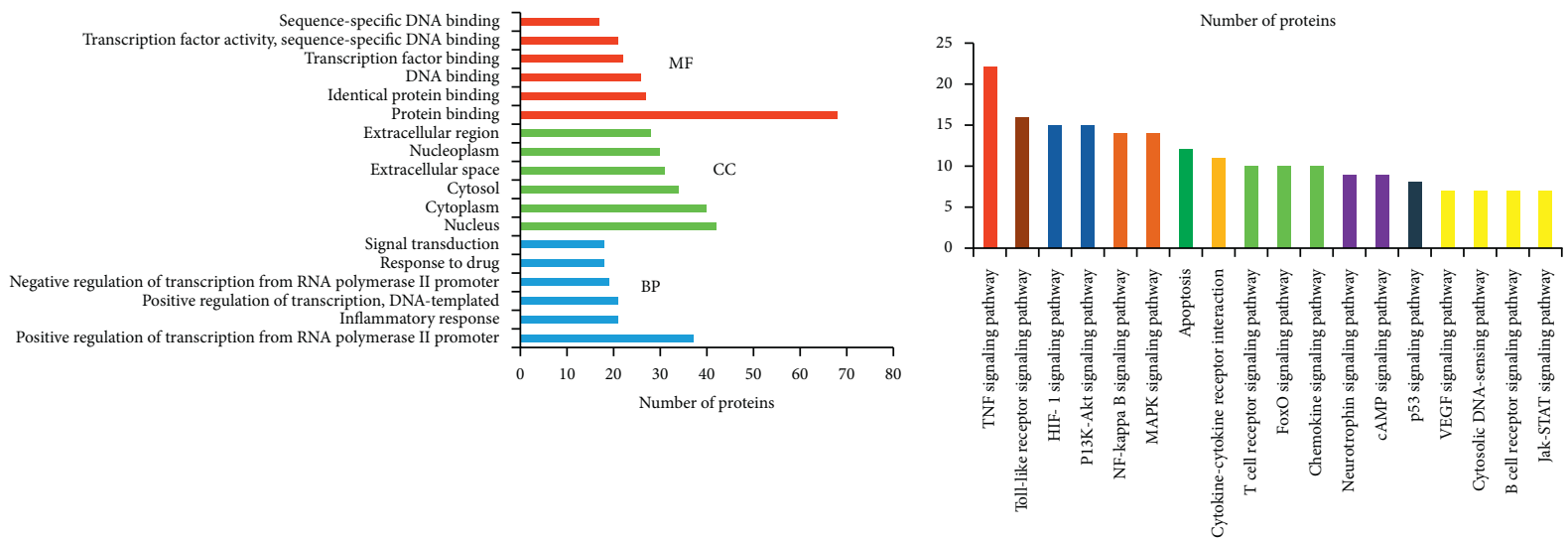

(a)

(b)

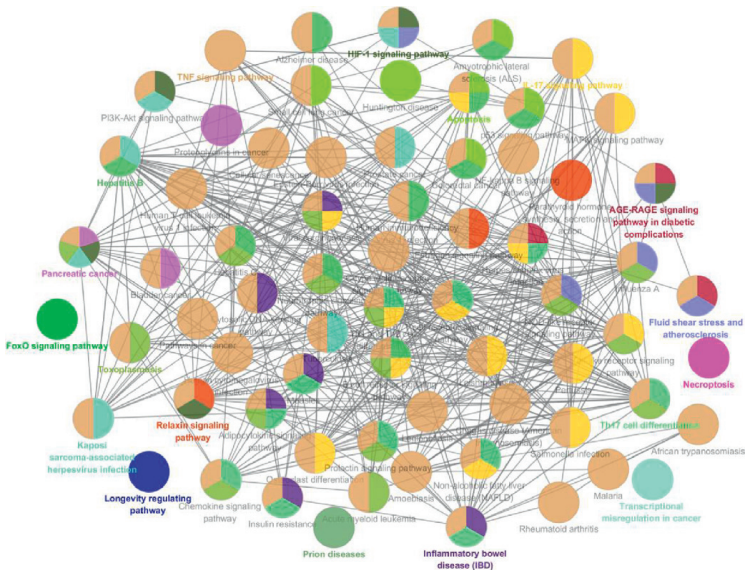

(c)

FIGURE 4: KEGG pathway and GO analysis by DAVID database. (a) GO analysis of candidate targets. Database showed the five remarkably enriched items in the biological processes (BP), cell component (CC), and molecular function (MF). (b) KEGG pathways of target genes. (c) Main functional annotation clusters by biocarta analysis.

TABLE 4: Functions of potential target genes based on KEGG pathway analysis.

\begin{tabular}{|c|c|c|c|c|}
\hline $\begin{array}{l}\text { Pathway } \\
\text { ID }\end{array}$ & Pathway classification & Term & Number of pathway genes & $p$ value \\
\hline hsa04668 & Signal transduction & TNF signaling pathway & $\begin{array}{c}\text { CCL2, CXCL10, CEBPB, FOS, JUN, JUNB, NFKBIA, RELA, } \\
\text { CREB1, CASP3, CSF2, ICAM1, IL1B, IL6, MMP9, MAPK1, } \\
\text { NFKB1, PIK3CG, PTGS2, RIPK1, SELE, TNF }\end{array}$ & $1 E-21$ \\
\hline hsa04620 & Immune system & $\begin{array}{l}\text { Toll-like receptor } \\
\text { signaling pathway }\end{array}$ & $\begin{array}{l}\text { CCL3, CXCL10, CD14, CD40, FOS, JUN, NFKBIA, RELA, } \\
\text { IL1B, IL6, MAPK1, NFKB1, PIK3CG, RIPK1, TLR4, TNF }\end{array}$ & $2.1 E-13$ \\
\hline hsa04066 & Signal transduction & HIF-1 signaling pathway & $\begin{array}{l}\text { BCL2, RELA, EPO, HIF1A, IGF1, IL6, MAPK1, NOS2, NOS3, } \\
\text { NFKB1, PIK3CG, SERPINE1, STAT3, TLR4, VEGFA }\end{array}$ & $9.2 E-13$ \\
\hline hsa04064 & Signal transduction & $\begin{array}{l}\text { NF-kappa B signaling } \\
\text { pathway }\end{array}$ & $\begin{array}{c}\text { ATM, BCL2, CD14, CD40, NFKBIA, RELA, ICAM1, IL1B, } \\
\text { NFKB1, PLAU, PTGS2, RIPK1, TLR4, TNF }\end{array}$ & $4.7 E-12$ \\
\hline hsa04210 & Cell growth and death & Apoptosis & $\begin{array}{c}\text { ATM, BCL2, NFKBIA, RELA, CASP3, CASP9, CYCS, NFKB1, } \\
\text { PIK3CG, RIPK1, TNF, TP53 }\end{array}$ & $3.4 E-11$ \\
\hline hsa04660 & Immune system & $\begin{array}{l}\text { T-cell receptor signaling } \\
\text { pathway }\end{array}$ & $\begin{array}{l}\text { FOS, JUN, NFKBIA, RELA, CSF2, IL10, MAPK1, NFKB1, } \\
\text { PIK3CG, TNF }\end{array}$ & 0.0000010 \\
\hline hsa04010 & Signal transduction & $\begin{array}{l}\text { MAPK signaling } \\
\text { pathway }\end{array}$ & $\begin{array}{l}\text { CD14, DDIT3, FOS, JUN, JUND, RELA, CASP3, HSPA1A, } \\
\text { IL1B MAPK1, MAPK3, NFKB1, TNF, TP53 }\end{array}$ & 0.0000023 \\
\hline hsa04115 & Cell growth and death & p53 signaling pathway & ATM, BBC3, CASP3, CASP9, CYCS, IGF1, SERPINE1, TP53 & 0.0000065 \\
\hline hsa04068 & Signal transduction & FoxO signaling pathway & $\begin{array}{l}\text { ATM, CAT, IGF1, IL10, IL6, MAPK1, PIK3CG, STAT3, SIRT1, } \\
\text { SOD2 }\end{array}$ & 0.0000120 \\
\hline
\end{tabular}


TABLE 4: Continued.

\begin{tabular}{|c|c|c|c|c|}
\hline $\begin{array}{l}\text { Pathway } \\
\text { ID }\end{array}$ & Pathway classification & Term & Number of pathway genes & $p$ value \\
\hline hsa04151 & Signal transduction & $\begin{array}{l}\text { PI3K-Akt signaling } \\
\text { pathway }\end{array}$ & $\begin{array}{c}\text { BCL2, JAK2, RELA, CREB1, CASP9, EPO, IGF1, IL6, MAPK1, } \\
\text { NOS3, NFKB1, PIK3CG, TLR4 }\end{array}$ & 0.0000140 \\
\hline hsa04722 & Nervous system & $\begin{array}{l}\text { Neurotrophin signaling } \\
\text { pathway }\end{array}$ & $\begin{array}{c}\text { BCL2, JUN, NFKBIA, RELA, CALM1, MAPK1, NFKB1, } \\
\text { PIK3CG, TP53 }\end{array}$ & 0.0000390 \\
\hline hsa04370 & Signal transduction & VEGF signaling pathway & CASP9, MAPK1, MAPK3, NOS3, PIK3CG, PTGS2, VEGFA & 0.0000440 \\
\hline hsa04623 & Immune system & $\begin{array}{l}\text { Cytosolic DNA-sensing } \\
\text { pathway }\end{array}$ & CXCL10, NFKBIA, RELA, IL1B, IL6, NFKB1, RIPK1 & 0.0000580 \\
\hline hsa04662 & Immune system & $\begin{array}{l}\text { B-cell receptor signaling } \\
\text { pathway }\end{array}$ & FOS, JUN, NFKBIA, RELA, MAPK1, NFKB1, PIK3CG & 0.0000890 \\
\hline hsa04062 & Immune system & $\begin{array}{l}\text { Chemokine signaling } \\
\text { pathway }\end{array}$ & $\begin{array}{c}\text { CCL2, CCL3, CXCL10, JAK2, NFKBIA, RELA, MAPK1, } \\
\text { NFKB1, PIK3CG, STAT3 }\end{array}$ & 0.0001500 \\
\hline hsa04060 & $\begin{array}{l}\text { Signaling molecules } \\
\text { and interaction }\end{array}$ & $\begin{array}{l}\text { Cytokine-cytokine } \\
\text { receptor interaction }\end{array}$ & $\begin{array}{c}\text { CCL2, CCL3, CXCL10, CD40, CSF2, EPO, IL1B, IL10, IL17A, } \\
\text { IL6, TNF }\end{array}$ & 0.0002500 \\
\hline hsa04024 & Signal transduction & cAMP signaling pathway & $\begin{array}{l}\text { FOS, JUN, NFKBIA, RELA, CREB1, CALM1, MAPK1, NFKB1, } \\
\text { PIK3CG }\end{array}$ & 0.0012000 \\
\hline hsa04630 & Signal transduction & $\begin{array}{l}\text { JAK-STAT signaling } \\
\text { pathway }\end{array}$ & JAK2, CSF2, EPO, IL10, IL6, PIK3CG, STAT3 & 0.0045000 \\
\hline
\end{tabular}

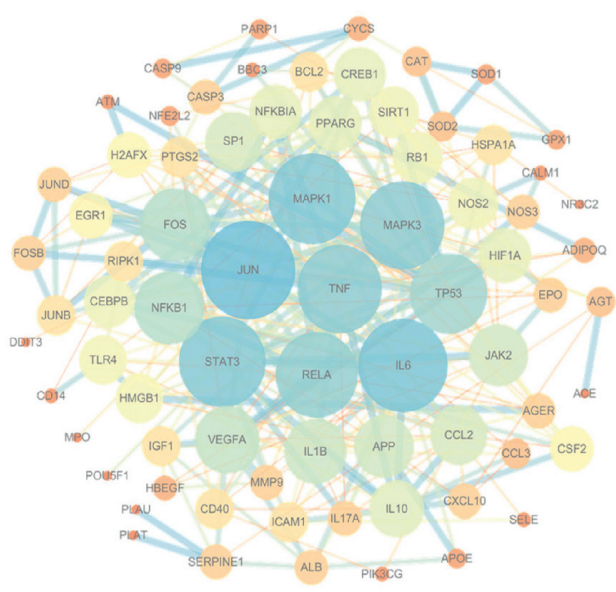

(a)

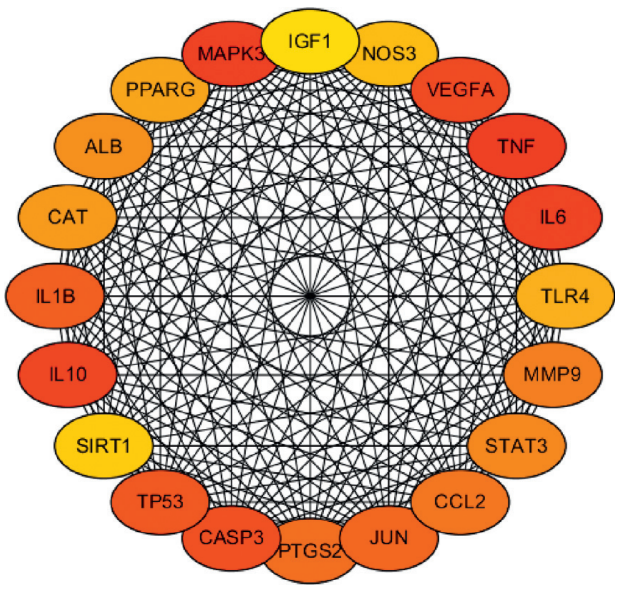

(b)

FIgURe 5: Protein-protein interaction (PPI) networks of active ingredients of HH for the treatment of cerebral ischemia. (a) Each node represents the relevant gene, the edge. Means line thickness indicates the strength of data support. (b) Center top 20 genes in the PPI network, the darker the color, the higher the score.

drugs, blood-activating drugs, and reinforcing Qi and activating blood drugs team were usually treated for CI with significant efficacy. A fewer side effects [23, 24] of these drugs could promote postischemic angiogenesis to recovery the cerebral blood flow and tissue perfusion, supporting the survival of neurons and neural progenitor cells and promoting long-term functional recovery $[25,26]$. HuangqiHonghua $(\mathrm{HH})$, a common reinforcing Qi and activating blood drugs team, has been used widely in clinical practice for treating Qi deficiency and blood stasis syndrome, especially, CI with Qi deficiency and blood stasis [7, 27]. Based on the available data, there are 276 compounds found in $\mathrm{HH}$ and categorized into flavonoids, lignans, terpene, sterols, triterpenoids, and their glycosides. After screening with $\mathrm{OB}$ and DL, we selected 21 compounds that were correlated with
78 human protein targets for CI. The top 10 key compounds related to more than five genes were found to be astragaloside IV, calycosin, formononetin, bifendate, isorhamnetin, hydroxysafflor yellow A, quercetin, luteolin, kaempferol, and beta-sitosterol. Uniformly, hydroxysafflor yellow A and astragaloside IV are in the list. Our previous study has indicated that $\mathrm{HH}$ could significantly ameliorate cerebral ischemia injury, and the mainly effective compounds are hydroxysafflor yellow A and astragaloside IV [13]. Moreover, in a recent study, astragaloside IV also has also been shown to possess anti-inflammatory and antioxidant activation potential [28]. Through the network analysis results, kaempferol has the maximum interactions with target genes, especially can attenuate neuroinflammation and blood brain barrier dysfunction to improve neurological deficits in 

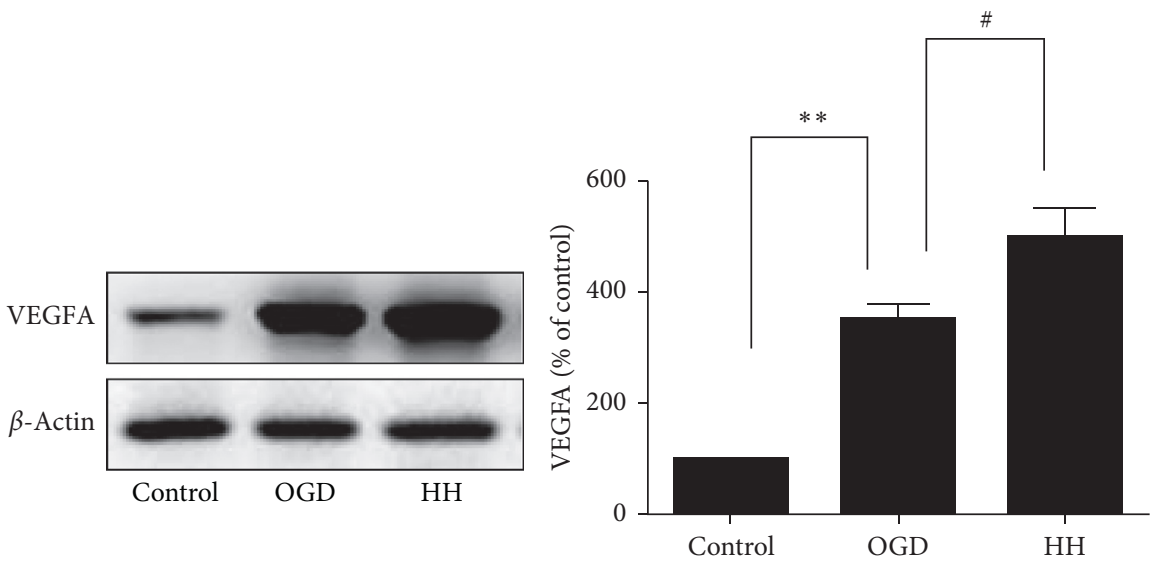

(a)
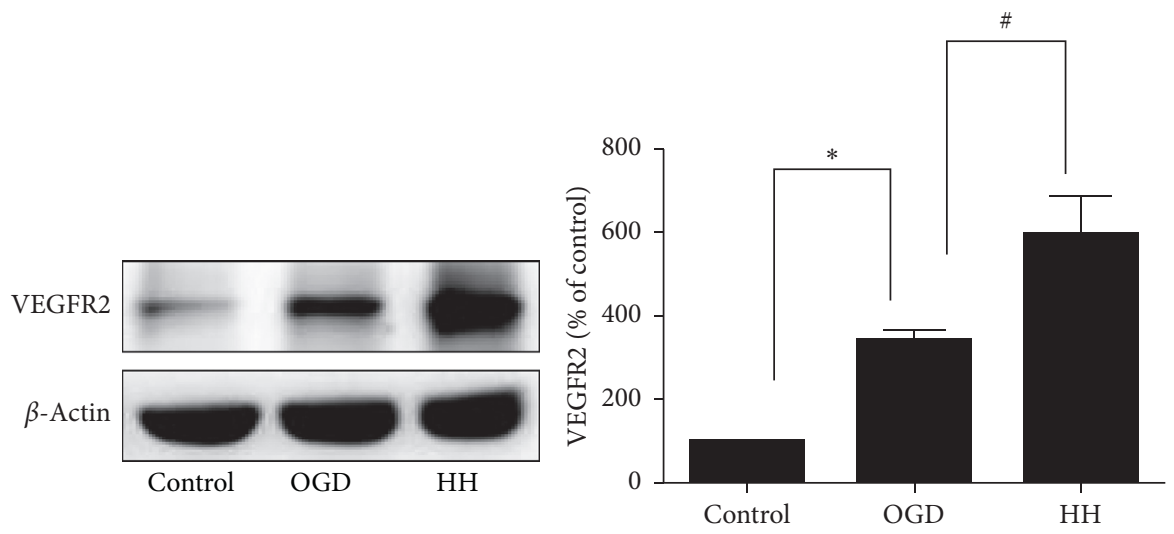

(b)

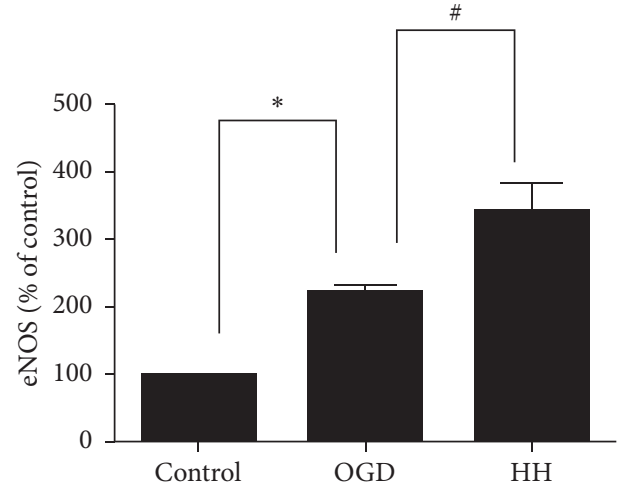

(c)

Figure 6: Effects of HH on the levels of VEGFA, VEGFR2, and eNOS in BMECs. $(* p<0.05$ and $* * p<0.01$ compared with the control group; $\# p<0.05$ compared with the OGD group).

cerebral I/R rats by regulating the $\mathrm{NK}-\kappa \mathrm{B}$ pathway [29]. However, further studies need to understand if kaempferol is the most important pharmacodynamics substance. Calycosin, which strikingly downregulates HUVEC TGF-betal, ICAM1, and RAGE expression [30], has antitumor, neuroprotective, anti-inflammatory, and proangiogenesis effects [31]. In vivo study also revealed quercetin to have antiinflammatory effects by inhibiting oxidative stress and cytokine production [32]. Formononetin exert its therapeutic effects by regulating these biological processes such as hormone metabolism, apoptosis, cell communication, and signal transduction [33]. These findings were consistent with the results from the active component target and indicate that the main components of $\mathrm{HH}$ are effective for treating target disease(s).

Angiogenesis is similar to "reinforcing qi to enrich blood, Dispelling stasis to promote regeneration" theory and regulated by angiogenesis inducers and inhibitors. Angiogenesis involves basilar membrane degradation, chemotactic migration, and proliferation of EC (endothelial cell) and 
EPC (endothelial progenitor cell). In the PPI system analysis, top 20 center genes were recognized, and among these genes, VEGFA and eNOS are highly correlated with angiogenesis. Over the few decades, vascular endothelial growth factors (VEGFs) and their receptors (VEGFRs) have been regarded as the principal drivers of angiogenesis and the development and maintenance of vascular system [34]. VEGFA (commonly known as VEGF) is the prototype member of the VEGF family of proteins, which stimulate angiogenesis in blood vascular endothelial cells via receptor-binding with VEGFR2 [35, 36]. Previous report suggests that overexpressed IncRNA ANRIL upregulates VEGF and promotes angiogenesis by activating NF- $\kappa \mathrm{B}$ pathway in CI rats [37]. Endothelial nitric oxide synthase (eNOS/NOS3) provides continuous local production of nitric oxide (NO), a crucial angiogenesis mediator and effector [38]. The increase in NO production via the upregulation of NOS3 by VEGF indicates that the angiogenic effect of VEGF seems to be mediated by NO [39]. Therefore, we verified the function of three essential genes (VEGFA, VEGFR2, and eNOS) using western blot assay, and the results show that VEGFA, VEGFR2, and eNOS were upregulated in BMECs with oxygen-glucose deprivation treatment with $\mathrm{HH}$, which is beneficial for angiogenesis.

According to the KEGG pathway analysis, these three target genes interact mainly with HIF-1 signaling pathway and VEGF signaling pathway. Low oxygenation concentrations in tissues (hypoxia) often trigger angiogenesis [40], which can be initiated independently of VEGF-related pathways, as well as lead to expression of multiple growth factors such as VEGF and NOS, via the HIF pathway [41]. HIF-regulated proangiogenic factors can increase vascular permeability, endothelial cell proliferation, migration, adhesion, and tube formation [42]. Therefore, we speculate that $\mathrm{HH}$ can regulate VEGFA, VEGFR2, and eNOS via the pathways associated with promote angiogenesis and alleviate cerebral ischemia injury. However, our current study still have some limitations, the compounds in the database may be incomplete, and it is incomprehensive that the vitro experiments only be used to confirm the results from the network. In the future, further studies require to design and implement a range of pharmacological experiment to investigate the possible pathway that controls proangiogenesis.

In summary, network pharmacology analysis of $\mathrm{HH}$ identified 2 herbs, 21 compounds, and 78 target gene-regulated major pathways associated with CI. The bioactive compounds in $\mathrm{HH}$ mainly participate in numerous signal transduction pathways such as TNF signaling pathway, tolllike receptor signaling pathway, HIF-1 signaling pathway, PI3K-Akt signaling pathway, and VEGF signaling pathway, and these important pathways may regulate anti-inflammatory, antiapoptotic, immune correlation, and antioxidative effects. Furthermore, through the pharmacological experiment, we predict that $\mathrm{HH}$ can regulate VEGFA, VEGFR2, and eNOS via the HIF-1 signaling pathway and VEGF signaling pathway to promote angiogenesis and alleviate cerebral ischemia injury.

\section{Abbreviations}

$\mathrm{HH}:$

CI:

TCM:

ADME:

BMECs:

OGD:

H- C-T-D

networks:

KEGG:

GO:

PPI:

Huangqi-Honghua herb pair

Cerebral ischemia

Traditional Chinese medicine

Absorption, distribution, metabolism, and excretion

Brain microvascular endothelial cells

Oxygen-glucose deprivation

Herb-compound-target-disease networks

Kyoto Encyclopedia of Genes

Genomes

Protein-protein interaction.

\section{Data Availability}

The data used to support the findings of this study are included within the article.

\section{Conflicts of Interest}

The authors declare that they have no conflicts of interest.

\section{Authors' Contributions}

C. J., L. L., W. K., S. J., W. J., W. A., and Y. Z. designed the experiments; C. J., L. L., W. K., Q. Y., D. J., and Z. C. conducted experiments and researched literature; C. J., L. L., C. J., and F. Z. collected and analyzed the data; C. J. and W. K. wrote the manuscript; C. J., L. L., W. K., D. J., and Y.Z. revised the manuscript. All authors commented on the results and approved the final manuscript. Jinyi Cao, Lu Lei, Kai Wang, and Jing Sun contributed equally to this work.

\section{Acknowledgments}

This research was funded by the National Natural Science Foundation of China (nos. 81503280, 81573549, 81603320, and 81603350).

\section{References}

[1] R. Lozano, M. Naghavi, K. Foreman et al., "Global and regional mortality from 235 causes of death for 20 age groups in 1990 and 2010: a systematic analysis for the global burden of disease study 2010," Lancet, vol. 380, no. 9859, pp. 2095-2128, 2012.

[2] S. K. Halder, H. Matsunaga, and H. Ueda, "Prothymosin alpha and its mimetic hexapeptide improve delayed tPA-induced brain damage following cerebral ischemia," Journal of Neurochemistry, vol. 153, no. 6, pp. 772-779, 2020.

[3] F. Ma, A. Morancho, J. Montaner, and A. Rosell, "Endothelial progenitor cells and revascularization following stroke," Brain Research, vol. 1623, pp. 150-159, 2015.

[4] Y. Li, L. Mao, Y. Gao, S. Baral, Y. Zhou, and B. Hu, "MicroRNA-107 contributes to post-stroke angiogenesis by targeting dicer-1," Scientific Reports, vol. 5, p. 13316, 2015.

[5] Q. Zhao, J. Hu, J. Xiang et al., "Intranasal administration of human umbilical cord mesenchymal stem cells-conditioned medium enhances vascular remodeling after stroke," Brain Research, vol. 1624, pp. 489-496, 2015. 
[6] W. K. Li, G. F. Wang, T. M. Wang et al., "Protective effect of herbal medicine Huangqi decoction against chronic cholestatic liver injury by inhibiting bile acid-stimulated inflammation in DDC-induced mice," Phytomedicine, vol. 62, p. 152948, 2019.

[7] Y. Zhang, J. Wu, S. Guo et al., "The clinical efficacy and safety of the Chinese herbal medicine astragalus (Huangqi) preparation for the treatment of acute myocardial infarction: a systematic review of randomized controlled trials," Medicine (Baltimore), vol. 98, no. 16, Article ID e15256, 2019.

[8] S. J. Yue, L. T. Xin, Y. C. Fan et al., "Herb pair DangguiHonghua: mechanisms underlying blood stasis syndrome by system pharmacology approach," Scientific Reports, vol. 7, p. $40318,2017$.

[9] L. Liu, J. A. Duan, Y. Tang et al., "Taoren-Honghua herb pair and its main components promoting blood circulation through influencing on hemorheology, plasma coagulation and platelet aggregation," Journal of Ethnopharmacology, vol. 139, no. 2, pp. 381-387, 2012.

[10] H. Chen, H. Song, X. Liu et al., "Buyanghuanwu decoction alleviated pressure overload induced cardiac remodeling by suppressing Tgf-beta/Smads and MAPKs signaling activated fibrosis," Biomedicine Pharmacotherapy, vol. 95, pp. 461-468, 2017.

[11] Z. Q. Zhang, J. Y. Song, Y. Q. Jia, and Y. K. Zhang, "Buyanghuanwu decoction promotes angiogenesis after cerebral ischemia/reperfusion injury: mechanisms of brain tissue repair," Neural Regeneration Research, vol. 11, no. 3, pp. 435-440, 2016.

[12] X. Liu, Y. Min, W. Gu, Y. Wang, and Y. Tian, "Buyanghuanwu Tang therapy for neonatal rats with hypoxic ischemic encephalopathy," International Journal of Clinical Experimental Medicine, vol. 8, no. 10, pp. 18448-18454, 2015.

[13] J. Cao, Z. Chen, Y. Zhu et al., "Huangqi-Honghua combination and its main components ameliorate cerebral infarction with Qi deficiency and blood stasis syndrome by antioxidant action in rats," Journal of Ethnopharmacology, vol. 155, no. 2, pp. 1053-1060, 2015.

[14] B. Boezio, K. Audouze, P. Ducrot, and O. Taboureau, "Network-based approaches in pharmacology," Molecular Informatics, vol. 36, no. 10, 2017.

[15] S. Li and B. Zhang, "Traditional Chinese medicine network pharmacology: theory, methodology and application," Chinese Journal of Naturals Medicines, vol. 11, no. 2, pp. 110-120, 2013.

[16] N. Wang, Y. Zheng, J. Gu et al., "Network-pharmacology-based validation of TAMS/CXCL-1 as key mediator of XIAOPI formula preventing breast cancer development and metastasis," Scientific Reports, vol. 7, p. 14513, 2017.

[17] Z. Dong, P. Zhao, M. Xu et al., "Astragaloside IV alleviates heart failure via activating PPARalpha to switch glycolysis to fatty acid beta-oxidation," Scientific Reports, vol. 7, no. 1, p. 2691, 2017.

[18] G. A. Huber, S. M. Priest, and T. P. Geisbuhler, "Cardioprotective effect of hydroxysafflor yellow a via the cardiac permeability transition pore," Planta Medica, vol. 84, no. 8, pp. 507-518, 2018.

[19] H. J. Fan, L. Yu, W. F. Jin et al., "Pharmacokinetics-pharmacodynamics correlation of protocatechuic aldehyde and hydroxysafflor yellow a alone or their combination use in rats with hyperlipidemia," Zhongguo Zhong Yao Za Zhi, vol. 42, no. 13, pp. 2564-2570, 2017.

[20] A. Y. Lee, W. Park, T. W. Kang, M. H. Cha, and J. M. Chun, "Network pharmacology-based prediction of active compounds and molecular targets in Yijin-Tang acting on hyperlipidaemia and atherosclerosis," Journal of Ethnopharmacology, vol. 221, pp. 151-159, 2018.

[21] X. Li, L. Wu, W. Liu et al., "A network pharmacology study of Chinese medicine Qi ShenYi Qi to reveal its underlying multicompound, multi-target, multi-pathway mode of action," PLoS One, vol. 9, no. 5, Article ID e95004, 2014.

[22] W. Wang, T. Liu, L. Yang et al., "Study on the multi-targets mechanism of triphala on cardio-cerebral vascular diseases based on network pharmacology," Biomedicine and Pharmacotherapy, vol. 116, Article ID 108994, 2019.

[23] J. Min, B. Mao, H. L. Jiang, T. Fan, and W. Zhou, "Effects of "reinforcing Qi and activating blood" on stable chronic obstructive pulmonary disease (Qi deficiency and blood stasis syndrome," Sichuan Da Xue Xue Bao Yi Xue Ban, vol. 45, no. 4, pp. 601-605, 2014.

[24] W. H. Zang, S. H. Yin, D. C. Tang, and B. B. Li, "Effect of medicines for activating blood and reinforcing Qi on angiogenesis in infarcted myocardium edge area of acute myocardial infarction model in rats," Zhongguo Zhong Yao Za Zhi, vol. 39, no. 5, pp. 901-906, 2014.

[25] R. Y. Lu, D. F. Luo, S. H. Xiao et al., "Kallikrein gene transfer induces angiogenesis and further improves regional cerebral blood flow in the early period after cerebral ischemia/ reperfusion in rats," CNS Neuroscience and Therapeutics, vol. 18, no. 5, pp. 395-399, 2012.

[26] Z. G. Zhang and M. Chopp, "Neurorestorative therapies for stroke: underlying mechanisms and translation to the clinic," Lancet Neurology, vol. 8, no. 5, pp. 491-500, 2009.

[27] K. Wang, J. Wu, X. Duan et al., "Comparative efficacy of Chinese herbal injections for angina pectoris: a Bayesian network meta-analysis of randomized controlled trials," Complementary Therapies in Medicine, vol. 43, pp. 208-217, 2019.

[28] W. Qian, X. Cai, Q. Qian et al., “Astragaloside IV protects endothelial progenitor cells from the damage of ox-LDL via the LOX-1/NLRP3 inflammasome pathway," Drug Design and Development and Therapy, vol. 13, pp. 2579-2589, 2019.

[29] W. H. Li, X. Cheng, Y. L. Yang et al., "Kaempferol attenuates neuroinflammation and blood brain barrier dysfunction to improve neurological deficits in cerebral ischemia/reperfusion rats," Brain Research, vol. 1722, p. 146361, 2019.

[30] Y. Xu, L. Feng, S. Wang et al., "Calycosin protects HUVECs from advanced glycation end products-induced macrophage infiltration," Journal of Ethnopharmacology, vol. 137, no. 1, pp. 359-370, 2011.

[31] J. Gao, Z. J. Liu, T. Chen, and D. Zhao, "Pharmaceutical properties of calycosin, the major bioactive isoflavonoid in the dry root extract of Radix astragali," Pharmaceutial Biology, vol. 52, no. 9, pp. 1217-1222, 2014.

[32] M. A. K. Abdelhalim, S. A. A. Moussa, and H. A. Y. Qaid, "The protective role of quercetin and arginine on gold nanoparticles induced hepatotoxicity in rats," International Journal of Nanomedicine, vol. 13, pp. 2821-2825, 2018.

[33] H. Xiao, X. Qin, J. Wan, and R. Li, "Pharmacological targets and the biological mechanisms of formononetin for Alzheimer's disease: a network analysis," Medical Science Monitor, vol. 25, pp. 4273-4277, 2019.

[34] S. Karaman, V. M. Leppanen, and K. Alitalo, "Vascular endothelial growth factor signaling in development and disease," Development, vol. 145, no. 14, 2018.

[35] M. Shibuya, S. Yamaguchi, A. Yamane et al., "Nucleotide sequence and expression of a novel human receptor-type 
tyrosine kinase gene (FLT) closely related to the FMS family," Oncogene, vol. 5, no. 4, pp. 519-524, 1990.

[36] M. Simons, E. Gordon, and L. Claesson-Welsh, "Mechanisms and regulation of endothelial of endothelial VEGF receptor signalling," Nature Review Molecular Cell Biology, vol. 17, pp. 611-625, 2016.

[37] B. Zhang, D. Wang, T. F. Ji, L. Shi, and J. L. Yu, "Overexpression of lncRNA ANRIL up-regulates VEGF expression and promotes angiogenesis of diabetes mellitus combined with cerebral infarction by activating NF-kappaB signaling pathway in a rat model," Oncotarget, vol. 8, no. 10, pp. 17347-17359, 2017.

[38] A. Aicher, C. Heeschen, C. Mildner-Rihm et al., "Essential role of endothelial nitric oxide synthase for mobilization of stem and progenitor cells," Nature Medicine, vol. 9, no. 11, pp. 1370-1376, 2003.

[39] M. Loebig, J. Klement, A. Schmoller et al., "Evidence for a relationship between VEGF and BMI independent of insulin sensitivity by glucose clamp procedure in a homogenous group healthy young men," PLoS One, vol. 5, no. 9, Article ID e12610, 2010.

[40] R. R. Ramjiawan, A. W. Griffioen, and D. G. Duda, "Antiangiogenesis for cancer revisited: is there a role for combinations with immunotherapy?," Angiogenesis, vol. 20, no. 2, pp. 185-204, 2017.

[41] B. L. Krock, N. Skuli, and M. C. Simon, "Hypoxia-induced angiogenesis: good and evil," Genes Cancer, vol. 2, no. 12, pp. 1117-1133, 2011.

[42] G. H. Fong, "Mechanisms of adaptive angiogenesis to tissue hypoxia," Angiogenesis, vol. 11, no. 2, pp. 121-140, 2008. 